OPEN ACCESS

Received: 04.01.2021

Accepted: 06.03.2021

Published: 15.03.2021

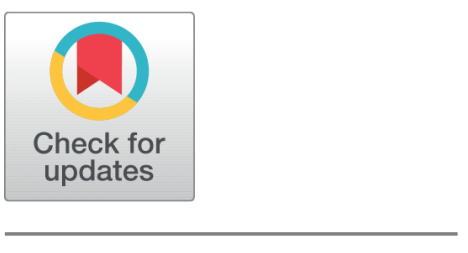

\section{Radiation and chemical reaction effects on unsteady MHD free convention mass transfer fluid flow in a porous plate}

\author{
Sweta Matta ${ }^{1,2 *}$, BalaSidduluMalga ${ }^{2 *}$, Lakshmi Appidi ${ }^{1,2}$, P Pramod Kumar ${ }^{2,3}$ \\ 1 Department of Mathematics, CMR Technical Campus, Kandlakoya, 501401, Telangana, India \\ 2 Department of Mathematics, GITAM University, Hyderabad, 502329, Telangana, India \\ 3 Department of Mathematics, B V Raju Institute of Technology, Narsapur, 502313, \\ Telangana, India
}

Citation: Matta S,

BalaSidduluMalga , Appidi L, Pramod Kumar P (2021) Radiation and chemical reaction effects on unsteady MHD free convention mass transfer fluid flow in a porous plate. Indian Journal of Science and Technology 14(8): 707-717. https://d oi.org/10.17485/IJST/v14i8.20

* Corresponding authors.

mswethamaths@gmail.com

drbsmalga@gmail.com

Funding: None

Competing Interests: None

Copyright: ( 2021 Matta et al. This is an open access article distributed under the terms of the Creative Commons Attribution License, which permits unrestricted use, distribution, and reproduction in any medium, provided the original author and source are credited.

Published By Indian Society for Education and Environment (iSee)

ISSN

Print: 0974-6846

Electronic: 0974-5645

\section{Abstract}

Objective: To study the effects of Radiation and chemical reaction on unsteady MHD free convection flow in a porous plate. Method: The problem is governed by coupled non-linear partial differential equations. The nondimensional equations of the problem have been solved numerically using Finite Element Method. Findings: The effects of governing parameters on the flow variables are discussed quantitatively with the help of graphs for the flow field, temperature field, concentration field, skin friction and Nusselt number. Novelty: The accuracy of the problem has been verified by comparing with the previous published work and the agreement between the results is excellent, which established confidence in the numerical results reported in this study.

Keywords: Radiation parameter; Chemical reaction; Eckert number; heat source parameter; micro polar fluid

\section{Introduction}

The role of thermal radiation on the flow and heat transfer process is major importance in the design of many advanced energy conversion systems operating at higher temperatures. Thermal radiation within the system is the result of emission by hot walls and the working fluid. Recently, considerable attention has also been focused on new applications of Magneto-hydrodynamics (MHD) and heat transfer such as metallurgical processing. Melt refining involves magnetic field applications to control excessive heat transfer rate. Other applications of MHD heat transfer include MHD generators, plasma propulsion in astronautics, nuclear reactor thermal dynamics and ionized- geothermal energy systems. Amo et al. ${ }^{(1)}$ studied the MHD convective flow over an inclined porous surface with variable suction and radiation effects. Prasad et al. ${ }^{(2)}$ studied the MHD flow of nano - fluid with radiation absorption. Ekakitie and $\mathrm{Amos}^{(3)}$ investigated the chemical reaction and heat source effects on MHD convection flows over an inclined surface. Pramod Kumar et al. ${ }^{(4)}$ interpreted the induced magnetic field on MHD. Kandasamy et al. ${ }^{(5)}$ interpreted the soret and duffer effects on free convective flow. Seddeek et al. ${ }^{(6)}$ analyzed the variable viscosity on mass transfer and MHD above a stretching sheet among chemical reaction. Mostafa et al. ${ }^{(7)}$ considered 
thermal results going on MHD through temperature dependent thickness. Ahmad et al. ${ }^{(8)}$ studied Thermophorosis results on micro polar fluid. Krishnendu et al. ${ }^{(9)}$ analyzed the special Effects of heat source on MHD. Bala Siddulu et al. ${ }^{(10)}$ has interpreted the suction results on flow of fluid accelerate Vertical Permeable Plate. Periasamy et al. ${ }^{(11)}$ has studied the results of chemical reaction by the side of a wedge in occurrence of suction. Anupam et al. ${ }^{(12)}$ analyzed the Chemical Reaction Effects on Nanofluid flows. Pal et al. ${ }^{(13)}$ interpreted the combine effects on MHD above plate in permeable media. Vijayaragavan et al. ${ }^{(14)}$ interpreted the Duffer and Chemical reaction effects on MHD convective Casson fluid flow over a vertical porous plate. Afzal Hoqet et al. ${ }^{(15)}$ have studied the error analysis of heat conduction Partial Differential Equations. Prabhakar reddy et al. ${ }^{(16)}$ investigated chemical reaction, Radiation effects lying on infinite isothermal vertical plate. Sweta et al. ${ }^{(17)}$ analyzed the heat absorption effects on MHD. Muralidharan et al. ${ }^{(18)}$ has study the Radiation effects on linearly accelerated plate with variable mass diffusion. Salawu et al. ${ }^{(19)}$ has studied inclined magnetic field with dissipation in a non-Darcy medium. Bala Siddulu et al. ${ }^{(20)}$ has explained heat generation, thermal diffusion results on moving plate.

The objective of the present work is to study the influence of radiation and chemical reaction effects on unsteady MHD free convection mass transfer fluid flow in porous plate. It has been noticed that the radiation and chemical reaction parameters had an effect on velocity profile, temperature profile, concentration profile. The governing partial differential equations are solved by Galerkin Finite Element Method. We have extended the problem of Balasiddulu ${ }^{(20)}$ in the presence of radiation parameter and the accuracy of present problem have been verified by comparing with theoretical solution of Balasiddulu ${ }^{(20)}$ through figures and the agreement between the results is excellent. This has established confidence in the numerical results reported in this paper.

\section{Mathematical Formulation}

An unsteady 2-dimensional coordinate system MHD free of convention in a porous plate with radiation and chemical reactions are considered. The flows are taken to be in $\mathrm{x}, \mathrm{y}$ directions to the plate. Here $\mathrm{x}$-axis represents the upward direction, $\mathrm{y}$-axis represents the normal to the plate. A homogeneous magnetic field considered in the direction upright to the plate. Reynolds numbers are less than 1 . The induced magnetic fields are insignificant and comparison by means of the considering magnetic fields. We considered that the fluids properties are stable. Also, there is a chemical reaction among the diffusing type and the fluid. The foreign mass presents in the flow of fluids are considered to be a small level and therefore Duffer, Soret results are unimportant. The above considerations, the governing equations written as

Continuity equation:

$$
\frac{\partial v^{*}}{\partial y^{*}}=0
$$

Momentum equation:

$$
\frac{\partial U^{*}}{\partial t^{*}}+V^{*} \frac{\partial U^{*}}{\partial y^{*}}=-\frac{1}{\rho} \frac{\partial p^{*}}{\partial x^{*}}+\left(v+V_{r}\right) \frac{\partial^{2} U^{*}}{\partial y^{* 2}}+g \beta_{f}\left(T-T_{\infty}\right)-v \frac{U^{*}}{K^{*}}+2 V_{r} \frac{\partial \omega^{*}}{\partial y^{*}}+g \beta_{c}\left(C-C_{\infty}\right)
$$

Angular momentum equation:

$$
\rho j^{*}\left(\frac{\partial \omega^{*}}{\partial t^{*}}+V^{*} \frac{\partial \omega^{*}}{\partial y^{*}}\right)=\frac{\partial^{2} \omega^{*}}{\partial y^{* 2}}
$$

Energy equation:

$$
\frac{\partial T}{\partial t^{*}}+V^{*} \frac{\partial T}{\partial y^{*}}=\alpha \frac{\partial^{2} T}{\partial y^{* 2}}+\mu\left(\frac{\partial U^{*}}{\partial y^{*}}\right)^{2}+\frac{S^{*}}{\rho C_{p}}\left(T-T_{\infty}\right)-\frac{1}{\rho C_{p}} \frac{\partial q_{r}}{\partial y^{*}}
$$

Diffusion equation:

$$
\frac{\partial C^{*}}{\partial t^{*}}+V^{*} \frac{\partial C^{*}}{\partial y^{*}}=D^{*} \frac{\partial^{2} C}{\partial y^{* 2}}-K_{1}^{*}
$$

fluid kinematic viscosity - $v$, fluid density- $\rho$, the coefficients about volume expansions meant for the concentration and the temperature are $\left(\beta_{c}, \beta_{f}\right)$, acceleration due to gravity- $\mathrm{g}$, fluid kinematic rotating viscosity - $\mathrm{V}_{r}$, micro-inertia density$\mathrm{j}^{*}$,empirical invariable called permeability about the porous medium - K, component of dimensional concentration - $\mathrm{C}^{*}$, 
temperature - T, chemical reaction parameter $\mathrm{K}_{1}{ }^{*}$, fluid dynamic viscosity- $\mu$, spin-gradient viscosity - $\gamma$, Dimensionless coordinate $\eta$, fluid thermal heated - $\alpha$ is the amount about mass diffusivity.

The boundary conditions are

$$
\left.\begin{array}{c}
U^{*}=0, T=T_{w}+\varepsilon\left(T_{w}-T_{\infty}\right) e^{n^{*} t^{*}}, \omega^{*}=-\frac{1}{2} \frac{\partial U^{*}}{\partial y^{*}}, C=C_{w}, \text { at } y^{*}=0 \\
U^{*} \rightarrow U_{\infty}^{*}=U_{0}\left(1+\varepsilon e^{n^{*} t^{*}}\right), T \rightarrow T_{\infty}, \omega^{*} \rightarrow 0, C \rightarrow C_{\infty}, \text { as } y^{*} \rightarrow \infty
\end{array}\right\}
$$

here $\mathrm{U}_{\infty}{ }^{*}$ - free stream velocity, $\mathrm{n}^{*}$ - dimensionless exponential index, $\varepsilon, \varepsilon$ A- undersized values fewer than $1, \mathrm{~V}_{0}$ - scale of suction velocity, $\mathrm{U}_{0}$ - scale of unbound stream velocity, A - constructive positive constant.

Outside the boundary layer, equation (2) becomes

$$
-\frac{1}{\rho} \frac{d p^{*}}{d x^{*}}=\frac{d U_{\infty}^{*}}{d t^{*}}+\frac{v}{K^{*}} U_{\infty}^{*}
$$

Using the following dimensionless quantities are

$$
\begin{gathered}
U=\frac{U^{*}}{U_{0}}, V=\frac{v^{*}}{V_{0}}, y=\frac{y^{*} V_{0}}{v}, U_{\infty}=\frac{U_{\infty}^{*}}{U_{0}}, \omega=\frac{v}{U_{0} V_{0}} \omega^{*}, t=\frac{t^{*} V_{0}^{2}}{v} \\
\theta=\frac{\left(T-T_{\infty}\right)}{\left(T_{W}-T_{\infty}\right)}, \quad C=\frac{\left(C^{*}-C_{\infty}\right)}{\left(C_{W}-C_{\infty}\right)}, n=\frac{n^{*} v}{V_{0}^{2}}, K=\frac{K^{*} V_{0}^{2}}{v^{2}}, j=\frac{V_{0}^{2}}{v^{2}} j^{*}, K_{1}=\frac{K_{1}^{*} v}{V_{0}^{2}} \\
S_{c}=\frac{v}{\mathrm{D}^{*}} \text { Schmidthnumber, } P_{r}=\frac{v}{\alpha} \quad \text { Prandtl number } \\
G_{r}=\frac{v g \beta_{f}\left(T_{w}-T_{\infty}\right)}{U_{0} V_{0}^{2}} \text { thermal Grashof number for heat } t \text { ransfer } \\
G_{m}=\frac{v g \beta_{f}\left(C_{w}-C_{\infty}\right)}{U_{0} V_{0}^{2}} \text { solutal Grashof number for mass transfer } \\
\gamma=\mu j^{*}\left(1+\frac{1}{2} \beta\right), \beta=\frac{\Lambda}{\mu}, \quad E_{c}=\frac{U_{0}^{2}}{C p\left(\mathrm{~T}_{\mathrm{w}}-\mathrm{T}_{\infty}\right)} \text { Eckert Number } \\
R=\frac{64 a v \bar{\sigma} \bar{T}_{\infty}^{3}}{\rho v_{0}^{2} C_{p}}, \\
S=\frac{S^{*} v}{\rho C_{p} V_{0}^{2}} \text { heat source parameter } \beta \text { is the dimensionless viscosity ratio }
\end{gathered}
$$

$\frac{\partial q_{r}}{\partial y^{*}}=-4 a \bar{\sigma}\left(\bar{T}_{\infty}^{4}-\bar{T}^{4}\right)$, Stefan Boltzmann constant $-\bar{\sigma}$, mean absorption - a. whereas the differences of the temperature in the flow accordingly undersized neglecting higher order terms then. Expressing $\bar{T}^{4}$ it is equal to the linear function of $\bar{T}$. Applying the method of the Taylor series expansion

By expanding $\bar{T}^{4}$ about the $\bar{T}_{\infty}$. Higher order terms are neglecting

$$
\bar{T}^{4}=4 \bar{T}_{\infty}^{3} \bar{T}-3 \bar{T}_{\infty}^{4}
$$

In examination of equation (6), (7), (8), (9) the equations (2), (3), (4) are reduces to the next non dimensional form are:

$$
\begin{aligned}
& \frac{\partial U}{\partial t}-\left(1+\varepsilon A e^{n t}\right) \frac{\partial U}{\partial y}=\frac{d U_{\infty}}{d t}+(1+\beta) \frac{\partial^{2} U}{\partial y^{2}}+G r \theta+\frac{1}{K}\left(U_{\infty}-U\right)+2 \beta \frac{\partial \omega}{\partial y}+G_{m} \\
& \frac{\partial \omega}{\partial t}-\left(1+\varepsilon A e^{n t}\right) \frac{\partial \omega}{\partial y}=\frac{1}{\eta} \frac{\partial^{2} \omega}{\partial y^{2}} \\
& \frac{\partial \theta}{\partial t}-\left(1+\varepsilon A e^{n t}\right) \frac{\partial \theta}{\partial y}=\frac{1}{\operatorname{Pr}} \frac{\partial^{2} \theta}{\partial y^{2}}+E_{c}\left(\frac{\partial U}{\partial y}\right)^{2}+S \theta+\frac{\operatorname{Pr} R}{4} \theta \\
& \frac{\partial C}{\partial t}-\left(1+\varepsilon A e^{n t}\right) \frac{\partial C}{\partial y}=\frac{1}{S c} \frac{\partial^{2} C}{\partial y^{2}}-K_{1} C
\end{aligned}
$$




$$
\text { Where, } \beta=\frac{V_{r}}{V}, \eta=\frac{\mu j^{*}}{\gamma}
$$

The resultant boundary conditions are

$$
\left.\begin{array}{rr}
U=0, \quad \theta=1+\varepsilon e^{n t}, & \omega=-\frac{1}{2} \frac{\partial U}{\partial y}, \mathrm{C}=1 \text { ony }=0 \\
U \rightarrow U_{\infty}, & \theta \rightarrow 0, \quad \omega \rightarrow 0, \quad C \rightarrow 0 \text { asy } \rightarrow \infty
\end{array}\right\}
$$

\section{Method of Solution}

The Galerkin FEM is applying to solve the equation (11), (12), (13) and (14) and above a 2 nodded linear component(e) $\left(y_{j} \leq y \leq y_{k}\right)$ is

$$
\begin{aligned}
& \int_{y_{j}}^{y_{k}} N^{T}\left[(1+\beta) \frac{\partial^{2} u}{\partial y^{2}}-\frac{\partial u}{\partial t}+\left(1+\varepsilon A e^{n t}\right) \frac{\partial u}{\partial y}+\frac{\partial U_{\infty}}{\partial t}+G r \theta+G m \varnothing+\frac{1}{k}\left(U_{\infty}-u\right)+2 \beta \frac{\partial \omega}{\partial y}\right] d y \\
& \int_{y_{j}}^{y_{k}} N^{T}\left[Z \frac{\partial^{2} u}{\partial y^{2}}-\frac{\partial u}{\partial t}+G \frac{\partial u}{\partial y}-\frac{1}{k} u+\frac{\partial U_{\infty}}{\partial t}+G r \theta+G m \varnothing+\frac{1}{k} U_{\infty}+2 \beta \frac{\partial \omega}{\partial y}\right] d y \\
& \int_{y_{j}}^{y_{k}}\left[Z \frac{\partial N}{\partial y} \cdot \frac{\partial u^{(e)}}{\partial y}-N^{T}\left(G \frac{\partial u^{(e)}}{\partial y}-\frac{\partial u^{(e)}}{\partial t}-\frac{1}{k} u^{(e)}+R\right)\right] d y=0 \\
& \text { where } G=\left(1+\varepsilon A e^{n t}\right), Z=(1+\beta), R=\left(G_{r} \theta+G_{c} C+\frac{1}{k} U_{\infty}+\frac{\partial U_{\infty}}{\partial t}+2 \beta \frac{\partial \omega}{\partial y}\right) \\
& N=\left[N_{j}, N_{k}, \phi\right]^{(e)}=\left[\begin{array}{l}
u_{j} \\
u_{k}
\end{array}\right] \\
& u^{(e)}=N . \phi^{(e)}, \quad N_{j}=\frac{y_{k}-y}{l^{(e)}}, N_{k}=\frac{y-y_{j}}{l^{(e)}}, l^{(e)}=y_{k}-y_{j}=h
\end{aligned}
$$

The element equations are given by

$$
\begin{aligned}
& \int_{y_{j}}^{y_{k}} Z\left[\begin{array}{cc}
N_{j}^{\prime} N_{j}^{\prime} & N_{j}^{\prime} N_{k}^{\prime} \\
N_{k}^{\prime} N_{j}^{\prime} & N_{k}^{\prime} N_{k}^{\prime}
\end{array}\right]\left[\begin{array}{c}
u_{j} \\
u_{k}
\end{array}\right] d y-G\left[\begin{array}{cc}
N_{j} N_{j}^{\prime} & N_{j} N_{k}^{\prime} \\
N_{k} N_{j}^{\prime} & N_{k} N_{k}^{\prime}
\end{array}\right]\left[\begin{array}{c}
u_{j} \\
u_{k}
\end{array}\right] d y+\left[\begin{array}{cc}
N_{j} N_{j} & N_{j} N_{k} \\
N_{k} N_{j} & N_{k} N_{k}
\end{array}\right]\left[\begin{array}{c}
\dot{u} \\
\dot{u_{k}}
\end{array}\right] d y+ \\
& \frac{1}{k}\left[\begin{array}{ll}
N_{j} N_{j} & N_{j} N_{k} \\
N_{k} N_{j} & N_{k} N_{k}
\end{array}\right]\left[\begin{array}{l}
u_{j} \\
u_{k}
\end{array}\right] d y-R\left[\begin{array}{l}
N_{j} \\
N_{k}
\end{array}\right] d y=0 \\
& \frac{Z}{l^{(e)}}\left[\begin{array}{cc}
1 & -1 \\
-1 & 1
\end{array}\right]\left[\begin{array}{l}
u_{j} \\
u_{k}
\end{array}\right]-\frac{G}{2}\left[\begin{array}{cc}
-1 & 1 \\
-1 & 1
\end{array}\right]\left[\begin{array}{l}
u_{j} \\
u_{k}
\end{array}\right]+\frac{1}{k} \frac{l^{(e)}}{6}\left[\begin{array}{cc}
2 & 1 \\
1 & 2
\end{array}\right]\left[\begin{array}{c}
u_{j} \\
u_{k}
\end{array}\right]+\frac{l^{(e)}}{6}\left[\begin{array}{cc}
2 & 1 \\
1 & 2
\end{array}\right]\left[\begin{array}{c}
\dot{u}_{J} \\
\dot{u}_{k}
\end{array}\right] d_{-} \\
& R \frac{l^{(e)}}{2}\left[\begin{array}{l}
1 \\
1
\end{array}\right]=0
\end{aligned}
$$

Where (.) denotes the differentiating with respective to $t$, we get $\left(y_{i-1} \leq y \leq y_{i}\right)$ and $\left(y_{i} \leq y \leq y_{i+1}\right)$, three element equations are assembling

$$
\begin{array}{r}
\frac{Z}{l^{(e)}}\left[\begin{array}{ccc}
1 & -1 & 0 \\
-1 & 2 & -1 \\
0 & -1 & 1
\end{array}\right]\left[\begin{array}{c}
u_{i-1} \\
u_{i} \\
u_{i+1}
\end{array}\right]-\frac{G}{2}\left[\begin{array}{ccc}
1 & 1 & 0 \\
-1 & 0 & 1 \\
0 & -1 & 1
\end{array}\right]\left[\begin{array}{c}
u_{i-1} \\
u_{i} \\
u_{i+1}
\end{array}\right]+\frac{1}{k} \frac{l^{(e)}}{6}\left[\begin{array}{ccc}
2 & 1 & 0 \\
1 & 4 & 1 \\
0 & 1 & 2
\end{array}\right]\left[\begin{array}{c}
u_{i-1} \\
u_{i} \\
u_{i+1}
\end{array}\right]+ \\
\frac{l^{(e)}}{6}\left[\begin{array}{ccc}
2 & 0 \\
1 & 4 & 1 \\
0 & 1 & 2
\end{array}\right]\left[\begin{array}{c}
\dot{u}_{i-1} \\
\dot{u}_{i} \\
\dot{u}_{i+1}
\end{array}\right]=R \frac{l^{(e)}}{2}\left[\begin{array}{c}
1 \\
2 \\
1
\end{array}\right]
\end{array}
$$


substitute row resultant to the node i to zero from difference schemes

$$
\begin{aligned}
& l^{(e)}=h \\
& \frac{z}{h^{2}}\left(-u_{i-1}+2 u_{i}-u_{i+1}\right)-\frac{G}{2 h}\left(-u_{i-1}+u_{i+1}\right)+\frac{1}{k} \frac{1}{6}\left(u_{i-1}+4 u_{i}+u_{i+1}\right)+\frac{1}{6}\left(\dot{u}_{i-1}+\right. \\
& \left.4 \dot{u}_{i}+\dot{u}_{i+1}\right)=R
\end{aligned}
$$

Crank-Nicolson method is applied to the above equation we get :

$$
A_{1} u_{i-1}^{n+1}+A_{2} u_{i}^{n+1}+A_{3} u_{i+1}^{n+1}=A_{4} u_{i-1}^{n}+A_{5} u_{i}^{n}+A_{6} u_{i+1}^{n}+12 k R
$$

Where, $R=G r T_{i}^{j}+G c C_{i}^{j}+\frac{1}{k} U_{\infty}+\frac{\partial U_{\infty}}{\partial t}+2 \beta \frac{\partial \omega}{\partial y}$

$R=G r T_{i}^{j}+G c C_{i}^{j}+\frac{1}{k} U_{\infty}+\frac{\partial U_{\infty}}{\partial t}$, consider $\xi=N$

Similarly, for the equation $(10),(11)$ following equations are obtained:

$$
\begin{aligned}
& B_{1} \theta_{i-1}^{n+1}+B_{2} \theta_{i}^{n+1}+B_{3} \theta_{i+1}^{n+1}=B_{4} \theta_{i-1}^{n}+B_{5} \theta_{i}^{n}+B_{6} \theta_{i+1}^{n} \\
& D_{1} C_{i-1}^{n+1}+D_{2} C_{i}^{n+1}+D_{3} C_{i+1}^{n+1}=D_{4} C_{i-1}^{n}+D_{5} C_{i}^{n}+D_{6} C_{i+1}^{n}+12 K S C R^{* *}
\end{aligned}
$$

Where, $R^{* *}=\operatorname{Sr} \frac{\partial^{2} \theta}{\partial y^{2}}$

$$
\begin{aligned}
& A_{1}=-6 r Z+3 G r h+S 1 k+2, A_{2}=12 r Z+4 S 1 k+8, A_{3}=-6 r Z-3 G r h+S 1 k+ \\
& 2, A_{4}=6 r Z-3 G r h-S 1 k+2, A_{5}=-12 r Z-4 S 1 k+8, A_{6}=6 r Z+3 G r h-S 1 k+2 \\
& \mathrm{~B} 1=2 \eta-6 \mathrm{r}+3 \mathrm{G} \eta \mathrm{rh}, \quad \mathrm{B} 2=8 \eta+12 \mathrm{r}, \quad \mathrm{B} 3=2 \eta-6 \mathrm{r}-3 \mathrm{G} \eta \mathrm{rh} \\
& \mathrm{B} 4=2 \eta+6 \mathrm{r}-3 \mathrm{G} \eta \mathrm{rh}, \quad \mathrm{B} 5=8 \eta-12 \mathrm{r}, \mathrm{B} 6=2 \eta+6 \mathrm{r}-3 \mathrm{G} \eta \mathrm{rh} \\
& C_{1}=-6 r+3 \mathrm{GPrrh}+2 \operatorname{Pr}+\operatorname{Pr} Q K, C_{2}=8 \operatorname{Pr}+12 r+4 \operatorname{Pr} Q K \\
& C_{3}=-6 r-3 \mathrm{GPrrh}+2 \operatorname{Pr}+\operatorname{Pr} Q K \\
& C_{4}=6 r-3 G \operatorname{Pr} r+2 \operatorname{Pr}-\operatorname{Pr} Q K, C_{5}=8 \operatorname{Pr}-12 r-4 \operatorname{Pr} Q K, \quad C_{6}=6 r+3 G P r r h+ \\
& 2 \operatorname{Pr}-\operatorname{Pr} Q K \\
& D_{1}=-6 r+3 G S c r h+2 S c+K 1 S c k, \quad D_{2}=12 r+8 S c+4 K 1 S c k \\
& D_{3}=-6 r-3 G S c r h+2 S c+K 1 S c k \\
& D_{4}=6 r-3 G S c r h+2 S c-K 1 S c k, \quad D_{5}=-12 r+8 S c-4 K 1 S c k \\
& D_{6}=6 r+3 G S c r h+2 S c-S c k
\end{aligned}
$$

$r=\frac{k}{h^{2}}$. Taking $\mathrm{h}=0.1, \mathrm{k}=0.001$ are considering for simplifications for the equations (19) to (21) and $\mathrm{h}, \mathrm{k}$ are mesh sizes along $\mathrm{y}$, $\mathrm{k}$ direction. Considering $\mathrm{i}=1(1) \mathrm{n}$ and using the initial, boundary condition (6) and (15).

$A_{i} X_{i}=B_{i}$, here $i=1,2,3 \ldots$

Here $A_{i}$ is the matrix of order $n$ and $X_{i}, B_{i}$ 's are column matrices having $n$-components. Thomas algorithm is used to solve the system of equations for Temperature, micro rotation, velocity and concentration and also arithmetical solutions used for these equations considered by c -programme to show the convergence and constancy of Galerkin Finite Element Method. The c-programme executed among small changes $h, k$ and no changes are observing in the standards of $u$, w, $\theta$ and $C$. For this reason we are observing that Galerkin Finite Element Method is convergent and stable.

Table 1. Sherwood number is given by $\mathrm{Sh}=-\left(\frac{\partial c}{\partial y}\right)$ at $\mathrm{y}=0$

\begin{tabular}{lllll}
\hline & $\mathrm{t}$ & Ec & Sc & \multicolumn{1}{l}{ Sh } \\
\hline 0.5 & 1.5 & 0.5 & 0.22 & 1.19953156 \\
0.8 & 1.2 & 0.6 & 0.5 & 1.59112000 \\
1.0 & 1.4 & 0.7 & 0.8 & 1.82498217 \\
1.0 & 1.5 & 0.7 & 0.8 & 1.82556152 \\
1.0 & 1.0 & 0.8 & 0.8 & 1.82556152 \\
1.0 & 1.5 & 1.0 & 1.0 & 1.933 .7602 \\
\hline
\end{tabular}


Table 2. Nusselt number is given by $\mathrm{Nu}=-\left(\frac{\partial \theta}{\partial y}\right)$ at $\mathrm{y}=0$

\begin{tabular}{llllll}
\hline$\beta$ & $\eta$ & $\mathrm{t}$ & $\mathrm{R}$ & $\mathrm{Ec}$ & $\mathrm{Nu}$ \\
\hline 0.4 & 0.5 & 1.0 & 1.0 & 0.5 & 0.51554173 \\
0.4 & 0.5 & 1.0 & 1.1 & 0.5 & 0.56152892 \\
0.4 & 0.5 & 1.0 & 1.2 & 0.7 & 0.58270884 \\
0.5 & 0.5 & 1.0 & 1.2 & 0.7 & 0.62856168 \\
0.5 & 0.5 & 1.0 & 1.4 & 0.8 & 1.10143113 \\
0.5 & 0.5 & 1.0 & 1.5 & 1.0 & 1.09877360 \\
0.5 & 0.5 & 1.0 & 1.5 & & 1.09867287 \\
\hline
\end{tabular}

Table 3. Skin friction number is given by $\tau=\left(\frac{\partial u}{\partial y}\right)$ at $\mathrm{y}=0$

\begin{tabular}{|c|c|c|c|c|c|c|}
\hline$\beta$ & $\eta$ & A & $\mathrm{Gr}$ & $\mathrm{Gc}$ & $\varepsilon$ & $\tau$ \\
\hline 0.4 & 0.6 & 1.0 & 2.0 & 2.0 & 0.001 & 0.34819758 \\
\hline 0.5 & 0.6 & 1.1 & 2.0 & 2.0 & 0.001 & 0.33404320 \\
\hline 0.5 & 0.6 & 1.3 & 2.2 & 2.2 & 0.001 & 0.33404320 \\
\hline 0.7 & 0.7 & 2.5 & 5.0 & 5.0 & 0.005 & 0.34162831 \\
\hline 0.7 & 0.7 & 2.0 & 5.0 & 5.0 & 0.001 & 0.30892715 \\
\hline 0.7 & 0.6 & 1.8 & 5.0 & 5.0 & 0.001 & 0.31479214 \\
\hline
\end{tabular}

\section{Results and Discussion}

The approximate values are obtained for concentration, temperature, angular velocity and velocity fields. The results of physical parameters Eckert number - (Ec), Prandtl number - (Pr), Schmidt number - $(\mathrm{Sc})$, the dimensionless viscosity $-(\beta)$, Grashof number meant for heat and mass transfer $-(\mathrm{Gr}, \mathrm{Gc})$, index number- $(\mathrm{n})$, the spin gradient viscosity $-(\gamma)$, chemical reaction parameter $-(\mathrm{K} 1)$, coefficient of gyro viscosity $-(\Lambda)$, time $-(\mathrm{t})$ considered through graphs.

The contribution of the time $-t$ lying on velocity profiles, angular velocity profiles is noticed in Fig 1a. and Fig 2a. We experiential that the velocity profiles raises and angular velocity profiles reduces.

It is seen that from Fig $1 \mathrm{~b}$. and Fig 2b.velocity increases and angular velocity increases with an result of index number-(n).

Fig $1 \mathrm{c}$, Fig $3 \mathrm{c}$ shown the viscous dissipation-(Ec) effects on the velocity, temperature results. We observe viscous dissipation increases then velocity results are raises, temperature results are reduces.

Fig 1.d., Fig2.c and Fig 4.c.observed that Chemical Reaction - $\left(\mathrm{K}_{1}\right)$ effects on velocity, angular velocity, concentration profiles. We observed that the velocity results raises all through the generative reaction $\left(K_{1}<0\right)$ and decrease throughout destructive reaction $\left(K_{1}>0\right)$, even though the reverse occurrence is observed used for micro- rotation. Concentration results are reduces.

Fig le.shown that Prandtl number -(Pr) increases then velocity fields are decreases.

Fig1f. Observing that velocity results improves with increasing in Gr, Gc.

The radiation parameter - $(\mathrm{R})$ e f f e $\mathrm{t}$ s o $\mathrm{n}$ the velocity profile, temperature profile, angular velocity profile are displays in the Fig 1.g, , Fig 3.e., Fig 2.g., Fig 4.d. We noticed that the radiation parameter raises, then the temperature and velocity profiles are raises and its observed that angular velocity are decreases.

Angular velocity increases with increases of $\eta$ in fig $2 \mathrm{~d}$.

Fig 3a. noticed that Prandtl number-(pr) increases then the temperature profiles are reduces.

fig 3 b.shown that effects of $n$ on the temperature fields.

Fig 3(d) noticed that suction parameter results on the temperature profile with raises on the suction parameter $S$ then the temperature decreases for a fixed $\eta$ and therefore thermal boundary layer thickness reduces.

Suction parameter raises then temperature profiles are reduces in Fig 3.d.

Fig 4.a. shown that growing in Schmidt number - $(\mathrm{Sc})$ then concentration profile decreases.

Fig 4 b.can be observed that decreasing in the concentration fields then increasing in index number $\mathrm{n}$. 
Graphs
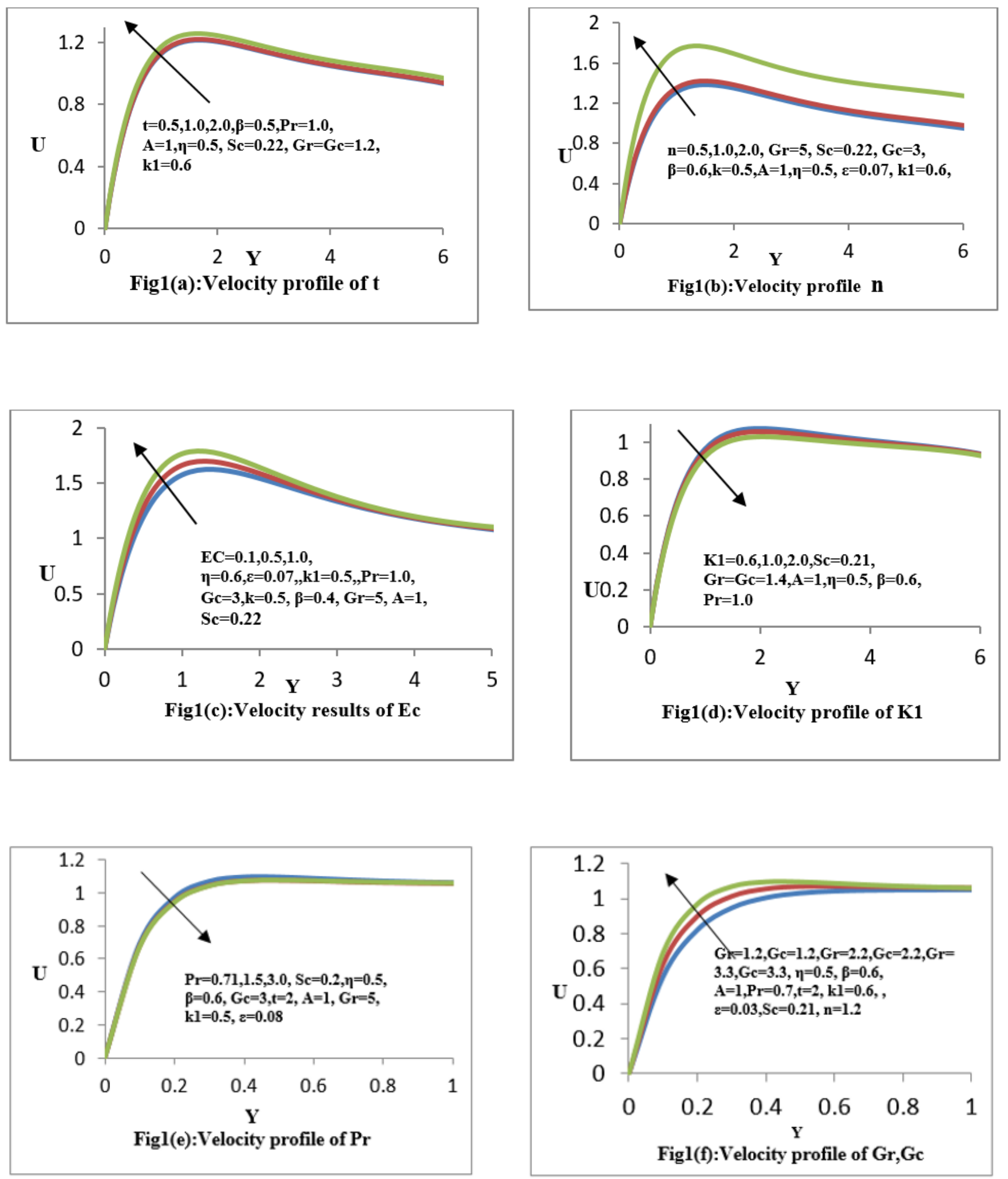


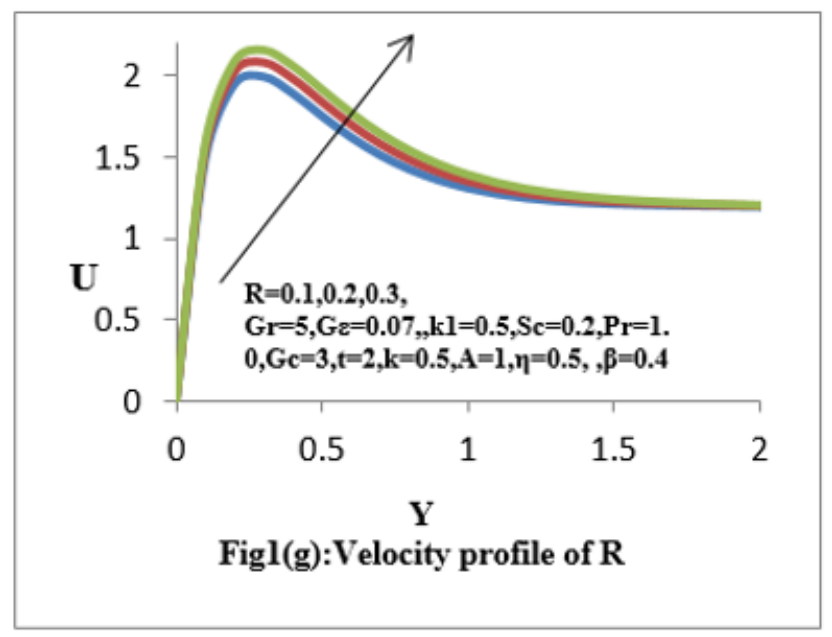

Fig 1.
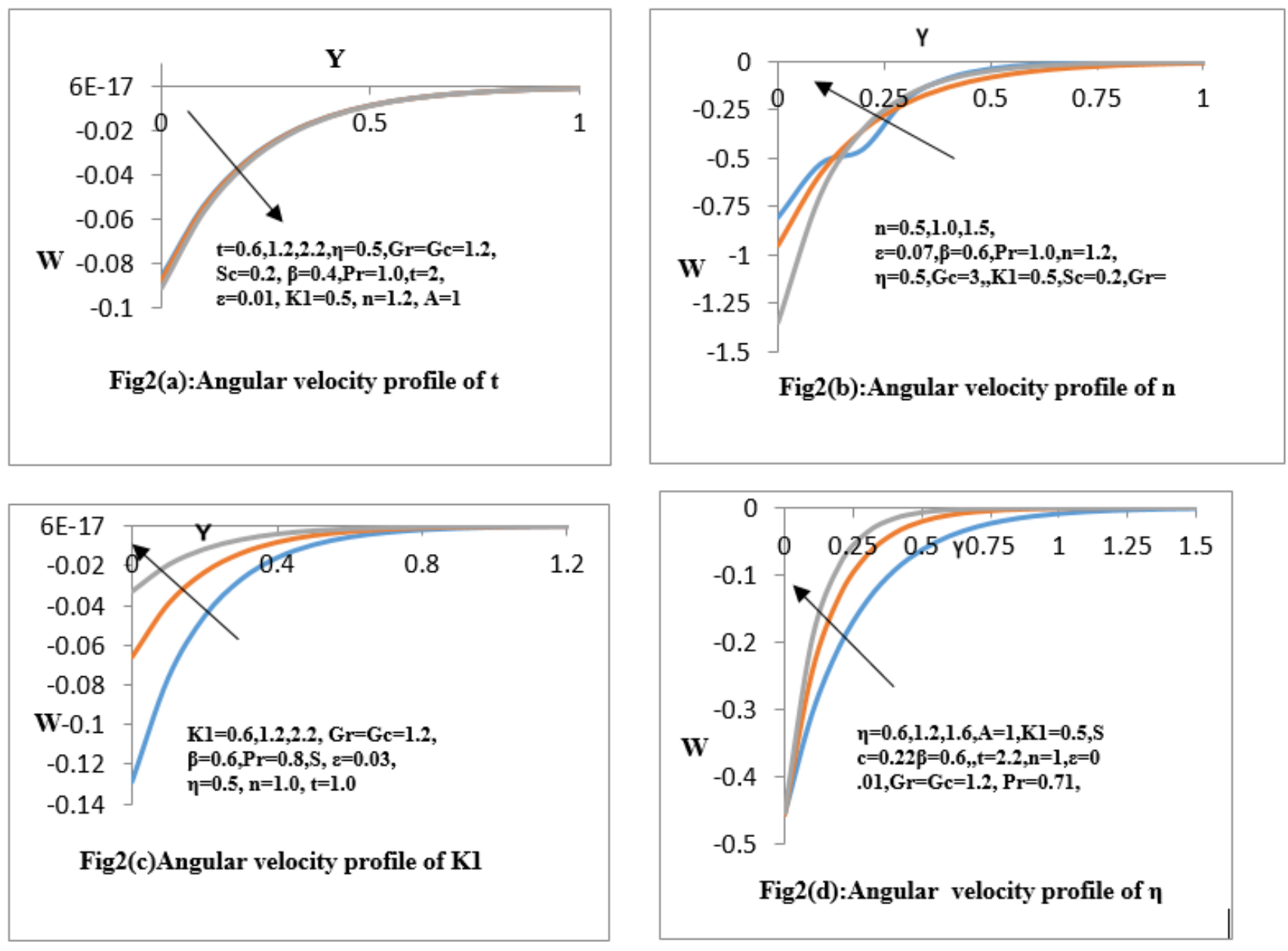


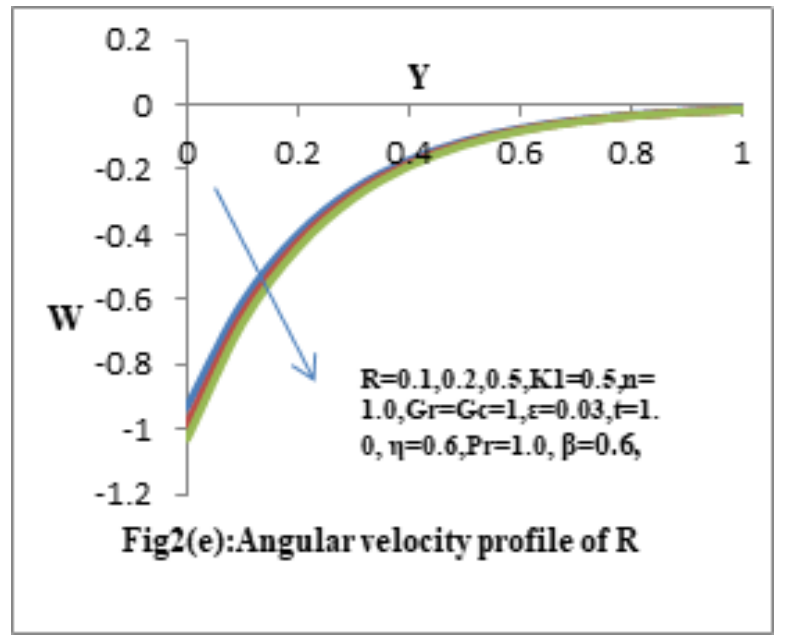

Fig 2.
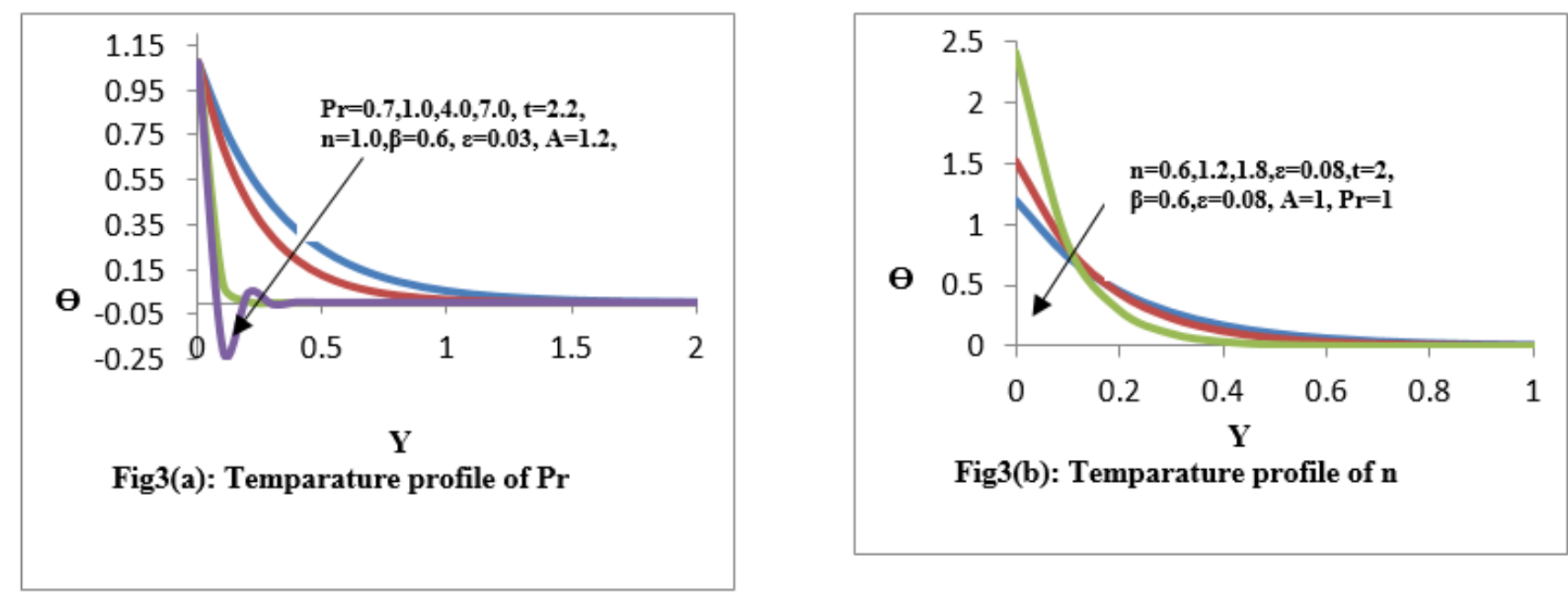

Fig3(b): Temparature profile of $n$
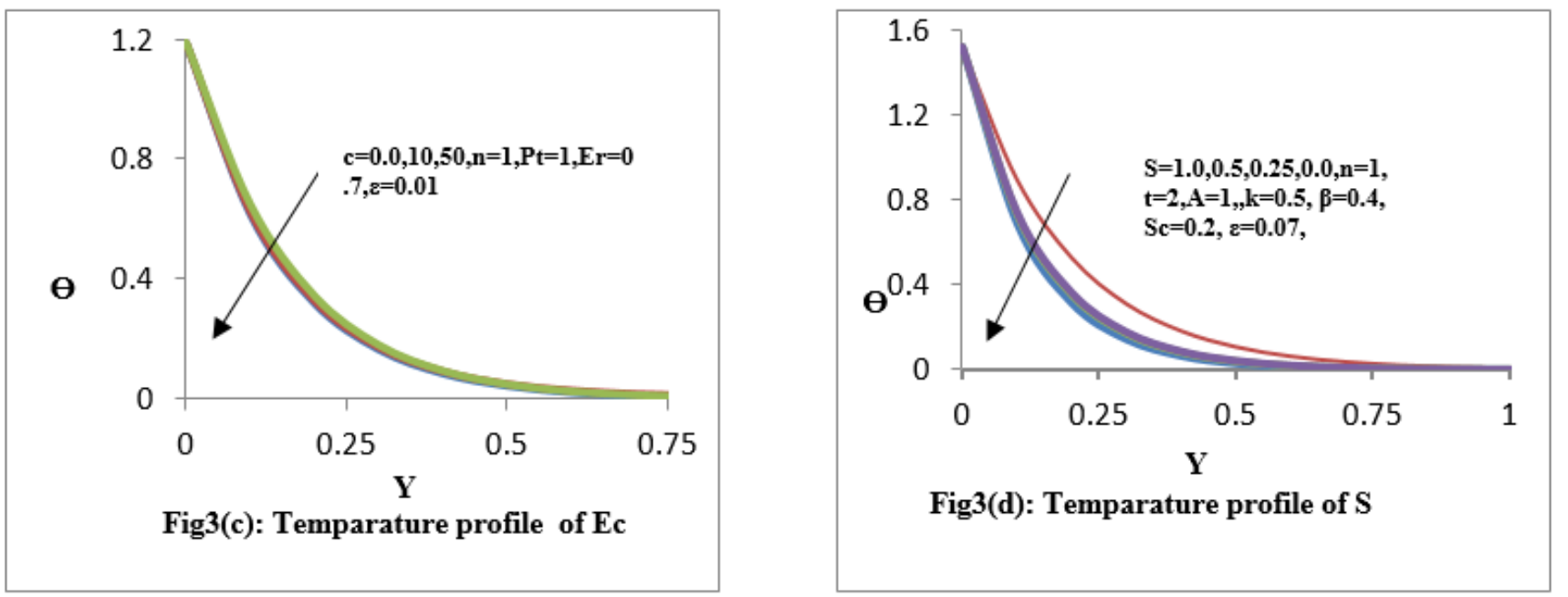


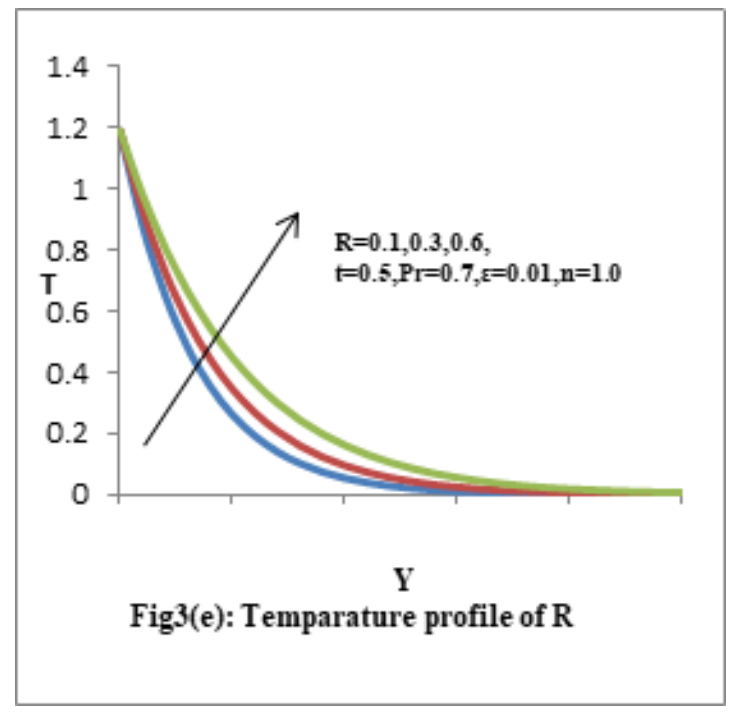

Fig 3.
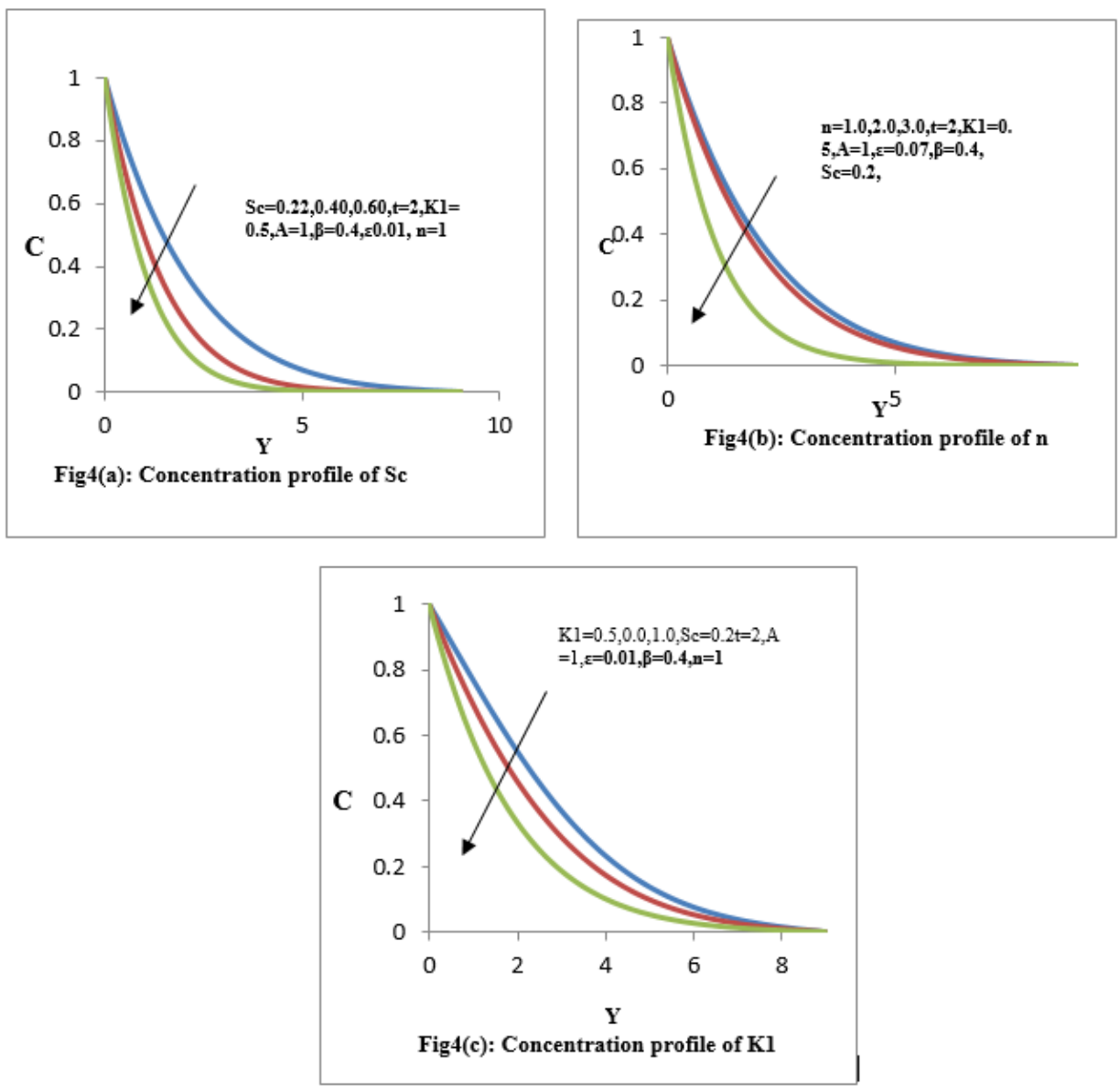

Fig 4. 


\section{Conclusion}

We studied the chemical reaction and heat source results on MHD. We applied finite element technique to solve the partial differential equations. The conclusions of the study are as follows:

- The velocity rises with the rising in Time, Eckert numbers, Thermal Grashof, Modified Grashof, Radiation parameter, Index number whereas the velocity reduces with the increases of chemical reaction parameter and Prandtl number.

- Increasing the temperature Radiation parameter increases while temperature decreases rising of Eckert number Prandtl

- Angular velocity profiles are increases with increasing of chemical reaction parameter, index number and angular velocity results are decreases with decreasing of radiation parameter and time.

- Schmidt parameter, chemical reaction parameter and index number increase when concentration profiles decrease.

\section{References}

1) Amos E, Omamoke E. MHD Free convective flow over an inclined porous surface with variable suction and radiation effects. International Journal of Applied Science and Mathematical Theory. 2018;4(3).

2) Prasad D, Kumar PK, S RVM, Varma SVK. Mass Transfer for the MHD Flow of Nano-Fluid with Radiation Absorption. Ain Shams Engineering Journal. 2016;9(4). Available from: https://doi.org/10.1016/j.asej.2016.04.016.

3) Omamoke E, Emeka A. The Impact of Chemical Reaction and Heat source on MHD Free Convection Flow over an Inclined Porous Surface. International Journal of Scientific and Research Publications (IJSRP). 2020;10(05):9-17. Available from: https://dx.doi.org/10.29322/ijsrp.10.05.2020.p10103.

4) Kumar PP, Malga BS, Matta S, Appidi L. Heat Transfer Reaction on a Viscous Dissipative Free Convective Radiating Stream over a Permeable Laminate within Presence of Induced Magnetic Field. International Journal of Innovative Technology and Exploring Engineering. 2020;9(3).

5) Kandasamy R, Hayat T, Obaidat S. Group theory transformation for Soret and Dufour effects on free convective heat and mass transfer with thermophoresis and chemical reaction over a porous stretching surface in the presence of heat source/sink. Nuclear Engineering and Design. 2011;241(6):2155-2161. Available from: https://dx.doi.org/10.1016/j.nucengdes.2011.03.002.

6) Seddeek MA, Almushigeh AA. Effects of radiation and variable viscosity on MHD free convective flow and mass transfer over a stretching sheet with chemical reaction. Applied Mathematics and Computation. 2010;5(1).

7) Mahmoud MAA. Thermal radiation effect on unsteady MHD free convection flow past a vertical plate with temperature-dependent viscosity. The Canadian Journal of Chemical Engineering. 2009;87(1):47-52. Available from: https://dx.doi.org/10.1002/cjce.20135.

8) Ahmad YB. Effects of Thermo phoresison Natural Convection boundary layer flow Of a Micropolar fluid. Thermal Science. 2010;14(1). Available from: https://doi.org/10.2298/TSCI1001171B.

9) Bhattacharyya K. Effects of heat source/sink on MHD flow and heattransfer over a shrinking sheet with mass suction. Chemical engineering Research Bulletn. 2011;15(1):12-17. Available from: https://doi.org/10.3329/cerb.v15i1.6524.

10) Malga BS, Kishan N. Viscous Dissipation Effects on Unsteady free convection and Mass Transfer Flow past an Accelerated Vertical Porous Plate with Suction. Advances in Applied Science Research. 2011;2(6).

11) Kandasamy R, Periasamy K, Prabhu KKS. Effects of chemical reaction, heat and mass transfer along a wedge with heat source and concentration in the presence of suction or injection. International Journal of Heat and Mass Transfer. 2005;48(7):1388-1394. Available from: https://dx.doi.org/10.1016/j. ijheatmasstransfer.2004.10.008.

12) Bhandari A. Radiation and Chemical Reaction Effects on Nanofluid Flow Over a Stretching Sheet. Fluid Dynamics \& Materials Processing. 2019;15(5):557582. Available from: https://dx.doi.org/10.32604/fdmp.2019.04108.

13) Pal D, Talukdar B. Combined effects of Joule heating and chemical reaction on unsteady magnetohydrodynamic mixed convection of a viscous dissipating fluid over a vertical plate in porous media with thermal radiation. Mathematical and Computer Modelling. 2011;54(11-12):3016-3036. Available from: https://dx.doi.org/10.1016/j.mcm.2011.07.030.

14) Vijayaragavan R, Karthikeyan S, Premalatha P. Heat and Mass Transfer on an Unsteady MHD mixed convective Casson fluid flow past a moving vertical porous plate with effects of the Dufour and Chemical reaction. International Journal of Mathematics Trends and Technology. 2019;65(2):117-129. Available from: https://doi.org/10.14445/22315373/IJMTT-V65I2P519.

15) Hoq SMA, Sulaeman E, Okhunov A. Error Analysis of Heat Conduction Partial Differential Equations using Galerkin's Finite Element Method. Indian Journal of Science and Technology. 2016;9(36). Available from: https://dx.doi.org/10.17485/ijst/2016/v9i36/102158.

16) Reddy BP. Radiation and Chemical Reaction Effects on Unsteady MHD Free Convection Parabolic Flow Past an Infinite Isothermal Vertical Plate with Viscous Dissipation. International Journal of Applied Mechanics and Engineering. 2019;24(2):343-358. Available from: https://dx.doi.org/10.2478/ijame2019-0022.

17) Matta S, Malga BS, Appidi L, Kumar PP. Finite element analysis of heat generation/absorption of Viscous Dissipation Effects on MHD Casson fluid flow over exponentially accelerated temperature with ramped surface concentration. AIP Conference Proceedings. 2020;p. 2269. Available from: https://doi.org/10.1063/5.0019762.

18) Muralidharan M, Muthucumaraswamy R. Radiation effects on linearly accelerated isothermal vertical plate with variable mass diffusion in the presence of magnetic field. Applied Mathematical Sciences. 2013;7(113):5645-5656. Available from: https://dx.doi.org/10.12988/ams.2013.38458.

19) Salawu SO, Dada MS, Radiative. Heat Transfer of Variable Viscosity and Thermal Conductivity Effects on Inclined Magnetic Field with Dissipation in a non- Darcy Medium. Journal of the Nigerian Mathematical Society. 2016;35(1). Available from: https://doi.org/10.1016/j.jnnms.2015.12.001.

20) Malga BS, Kishan N. Finite element analysis for unsteady MHD heat and mass transfer free convection flow of polar fluids past a vertical moving porous plate in a porous medium with heat generation and thermal diffusion. Journal of Naval Architecture and Marine Engineering. 2014;11(1):69-82. Available from: https://dx.doi.org/10.3329/jname.v11i1.12844. 